Résumés des conférences et travaux

\title{
Méthodes de la prosopographie à l'époque contemporaine
}

Isabelle Parizet

\section{OpenEdition}

\section{Journals}

Édition électronique

URL : https://journals.openedition.org/ashp/740

DOI : 10.4000/ashp.740

ISSN : 1969-6310

Éditeur

Publications de l'École Pratique des Hautes Études

\section{Édition imprimée}

Date de publication : 1 octobre 2009

Pagination : 278-286

ISSN : 0766-0677

\section{Référence électronique}

Isabelle Parizet, « Méthodes de la prosopographie à l'époque contemporaine », Annuaire de l'École

pratique des hautes études (EPHE), Section des sciences historiques et philologiques [En ligne], 140 | 2009, mis en ligne le 20 octobre 2009, consulté le 06 juillet 2021. URL : http://journals.openedition.org/ashp/ 740 ; DOI : https://doi.org/10.4000/ashp.740 


\title{
MÉTHODES \\ DE LA PROSOPOGRAPHIE À L'ÉPOQUE CONTEMPORAINE
}

\author{
Maître de conférences : $\mathrm{M}^{\mathrm{me}}$ Isabelle PARIZET
}

Programme de l'année 2007-2008 : I. Les différentes spécialités de la sculpture de façades. II. Notices prosopographiques sur les sculpteurs de façades et ornemanistes au XIX ${ }^{e}$ s.

En analysant les sources, il est apparu que sur un chantier se succédaient « tailleur de pierres, metteur aux points, praticien, sculpteur d'ornements, ornemaniste, figuriste, animalier, statuaire, graveur de lettres ...» Spécialistes d'un savoir-faire, d'une technique ou d'un motif, ils intervenaient ponctuellement à une étape bien particulière du travail et exécutaient une tâche limitée dans le temps et l'espace. Afin de faire une étude chronologique rigoureuse et scientifique de leurs interventions, le séminaire a analysé en priorité les fonds d'archives publiques (plus complets que ceux des travaux privés). Ont été consultées les séries des édifices diocésains (Arch. nat. : $\mathrm{F}^{19}$ ), celles des Bâtiments civils (Ibidem, $\mathrm{F}^{21}$ ), du Louvre (Ibidem, 64AJ), du nouvel Opéra (Ibidem, $\mathrm{AJ}^{13}$ ), des expositions universelles (Ibidem, $\mathrm{F}^{12}$ ) et des édifices municipaux (Arch. Paris : V.M).

Le choix des pierres revient à l'administration de tutelle (sauf pour les marbres désignés par le statuaire lui-même). Selon leur fonction et leur emplacement définitifs, la sélection se fait en carrière, en tenant compte de leur composition, de leur consistance ou tonalité et de leur prix (souvent la proximité de Paris enlève la décision). Le carrier conseille les roches les plus résistantes (mais plus difficiles à travailler) pour les soubassements, rappelle que les calcaires sédimentés en couches horizontales doivent être redressés et exploités « à contre-lit », ou que les marbres disposés en strates successives sont plus ou moins homogènes de densité ou de couleur. Les blocs ne doivent présenter aucun défaut de structure comme une zone poreuse ou disparate, une fracture (même microscopique) ou un fil qui risqueraient de les fragiliser alors que taches, nœuds ou petits trous peuvent toujours être polis ou rebouchés. Dans les archives de la construction du nouvel Hôtel de Ville, une liste définit les « Travaux de sculpture de façades à prévoir » : pour chaque étage, les motifs y sont désignés, précisant leur nombre et leurs dimensions (Arch. Paris : V.1M $\mathbf{M}^{2}$ 29). Il est intéressant de noter que si les sculpteurs ne sont pas encore nommés (seuls certains sont proposés au crayon dans la colonne des « Observations »), l'administration a déjà choisi la nature de la pierre. Ainsi, pour le « pavillon d'angle côté quai de la façade Lobau, le rez-de chaussée sera en pierre dure de Charentenay », au $1^{\text {er }}$ étage les «chapiteaux et tympans en Courson et le couronnement des angles sous écusson, consoles et feuilles d'angle et la grande lucarne en Lavaux ».

Le tailleur de pierres prépare grossièrement le bloc et l'allège au maximum pour le transport en enlevant le bousin et en l'équarrissant pour lui donner une première forme 
de quadrilatère aux surfaces planes. Pendant ce temps, l'administration demande au sculpteur de lui indiquer l'adresse de livraison et les dimensions qu'il souhaite lui voir donner : le carrier affine alors son travail en épannelant la pierre suivant les mesures indiquées et lui donne une première forme grossière du motif final, très chanfreinée.

La livraison peut se faire sur le chantier ou à l'atelier selon que le travail de taille sera fait «sur le tas » (la pierre est sculptée in situ) ou « avant la pose » (au sol devant l'édifice ou en atelier). Dans le premier cas, comme les blocs épannelés sont directement installés dans l'architecture, le sculpteur est plus à même de juger des jeux d'ombre et de lumière, d'adapter l'exécution de motifs ou de respecter les divisions de l'appareil (il peut éviter par exemple que les joints ne coupent les ornements) mais, par contre, certains angles deviennent inaccessibles. Dans le second, le travail est facilité par un accès aisé aux quatre faces mais sera fragilisé par la mise en place. Pour les statues de la balustrade du nouveau Louvre, un «État des dépôts » faits par Fouillère, carrier installé à Conflans-Sainte-Honorine, recense quatorze livraisons en ateliers entre le 22 juin et le 6 août 1854 (Arch. nat. : 64AJ 186). Par contre, à l'Opéra, les carriers déposent tous les blocs sur le chantier, puis les transportent dans les différents ateliers. Lors de toute remise, un « billet de réception » comprenant le nom du sculpteur, l'adresse, le numéro de commande et les mesures, est signé par le praticien ou un de ses adjoints : ainsi Milan signe pour le statuaire Laurent :

«Commande n ${ }^{\circ} 39$. Laurent, statue de Boucher. Reçu de l'Hôtel de Ville le bloc de pierre des dimensions suivantes : $2.50 \times 1.00 \times 0.70$. Paris le 20 octobre $1880 . \mathrm{A}^{\text {dre }}$ Milan sculpteur, boulevard de Grenelle $65 »$ (Arch. Paris : V.1M² 33).

Le passage de l'octroi s'avère complexe et onéreux pour les ateliers installés dans les communes entourant Paris (essentiellement Montrouge et Montmartre). Ainsi, toujours à l'Hôtel de Ville, le praticien Goemans est installé au 35, rue du Petit Parc à Montrouge. Lors de la livraison, l'architecte M. Ballu prévient qu'il « ne veut pas expédier aussi loin car cela occasionnerait des frais d'octroi ». Une solution est envisagée avec le prêt d'un atelier à l'Hôtel de Ville. Finalement, la pierre sera remise à Montrouge le 11 juillet 1881, après avoir obtenu l'exemption des frais d'octroi (Ibidem, V.1M $\mathrm{M}^{2} 36$, commande $\mathrm{n}^{\circ} 120$ du 19 novembre 1880 au statuaire Henri Plé, statue de Daubigny, niche du $1^{\text {er }}$ étage de la façade Lobau).

Á réception du bloc et avant de se mettre au travail, le sculpteur l'inspecte attentivement et la palpe de ses instruments : P. Aubry n'engage sa signature qu' "après avoir examiné le modèle de chapiteau..., après avoir touché à l'outil le marbre blanc épannelé et mouluré dans lequel doit être taillé le dit chapiteau » (Louvre, Arch. nat. : 64AJ 189). Car, en cas d'accident durant le transport ou de la découverte d'un fil, un constat doit être établi par un inspecteur afin de pouvoir en recommander une autre : ainsi, pour la statue de Marivaux, Albert-Lefeuvre prévient la direction des Travaux :

« Il y a dans la pierre que vous m'avez faite envoyer un fil qui traverse la tête et qui parait entièrement détaché. Je crois qu'il faudrait un autre morceau de pierre. Voudriezvous envoyer constater ce fait... » (Hôtel de Ville, Arch. Paris : V.1M²33).

Cependant, quelque soit le soin mis par les sculpteurs pour analyser leur bloc lors de la livraison, ils restent toujours à la merci de dégradations spontanées dues à la 
structure de la roche (zone hétérogène), aux intempéries (pluie, gel) ou à des facteurs liés à la construction elle-même (contraintes physiques) ou à son emplacement (érosion chimique).

Les statuaires sont les professionnels qui jouissent de la plus grande notoriété. Sur les façades, ils interviennent pour l'exécution des cariatides, frontons, groupes, motifs de couronnement, bas-reliefs et statues. Qualifiés d'une compétence et d'un savoirfaire reconnus par l'Académie, dès leur retour de Rome, ils sont rapidement gratifiés de commandes avantageuses. Les sujets en sont historiques, figurés ou allégoriques. Toutefois, leur formation très théorique à l'École des beaux-arts ne les a que peu préparés à la pratique : ils se font donc seconder par des praticiens, artisans experts dans l'art de manier le ciseau.

Pour traduire dans la pierre le modèle (dessin ou plâtre) accepté à la fois par l'administration et le sculpteur, plusieurs outils de report sont à la disposition des praticiens : la plus ancienne, la « caisse à baguettes » conçue par Léonard de Vinci le place dans une sorte de caisson dans lequel sont enfoncées des tiges graduées qui permettent d'en prendre les dimensions. D'autres appareils reportent directement les différentes cotes sur le bloc : la « machine à mise aux points » de Gatteaux, le «système à trois compas » qui transpose les mesures selon une échelle de conversion, et enfin la « machine pantographe », reprise et améliorée par Achille Colas et Frédéric Sauvage (puis Ferdinand Barbedienne) qui reproduit le modèle par réduction mécanique.

La plupart des praticiens ont été formés à ce travail sur le tas par leur chef d'atelier : ils ont souvent commencé comme tailleur de pierres, puis ont été remarqués pour leur adresse. Ils travaillent sous la direction du statuaire et sont attachés à un ou plusieurs sculpteurs : à l'Hôtel de Ville, Michel Dekeyser est embauché à la même époque par J. M. H. Chevalier, J. B. D. Dupuis et F. Leenhoff (arrêté du 19 novembre 1880). La difficulté consiste à exécuter simultanément quatre commandes dont les blocs sont livrés les 27 juin (Leenhoff), 8 juillet (Chevalier), et 28 et 29 septembre (les deux derniers pour Dupuis, cf. Arch. Paris : V.1M $1 \mathrm{M}^{2} 31$, V.1M 32 et V.1 $\mathrm{M}^{2} 33$ ). Quant la mise aux points est terminée, le statuaire (prévenu par son praticien) demande à l'administration ou à l'architecte de passer à l'atelier :

« J'ai l'honneur de vous informer que la mise aux points de ma statue de Catinat est entièrement terminée. Je vous serai obligé si vous vouliez bien venir chez mon praticien afin d'examiner son travail » demande Massoule à l'agence des travaux de l'Hôtel de Ville (Ibidem, V.1M² 35).

Il est important de noter ici que d'une part la statue est toujours « chez le praticien », et que de l'autre, le statuaire n'y a toujours pas touché puisqu'il suggère d'examiner «son » travail.

Guidé par les repères laissés par les machines, le praticien poursuit le travail à l'aide d'outils plus coupants (pointe, tranchant, gradine ou ciseau). Il suit les observations de l'inspecteur chargé de surveiller régulièrement son travail et les instructions du statuaire. Quant celui-ci est satisfait, il se met lui-même à la tâche, apporte quelques corrections, puis demande au praticien de faire les ultimes retouches, de ravaler toutes les saillies accidentelles, d'effacer les traces d'outil, de raser les points de reports 
(quelques uns ont été oubliés et sont encore visibles au pied du groupe allégorique de François Jouffroy, L'Harmonie, $1^{\text {er }}$ à gauche sur la façade principale de l'Opéra) et de commencer à polir les surfaces.

Parmi ces praticiens, on peut constater la présence de plusieurs italiens venus à Paris lors de l'ouverture d'un des grands chantiers publics et qui, finalement, s'y installent définitivement (cf. Agostini, Bartelletti, Buzzonnetti, Casini, Ceribelli, Cochi, Musetti, pour n'en citer que quelques uns). Malgré la xénophobie en cours, leur capacité est rapidement reconnue et souhaitée par certains chefs d'atelier. Le cas d'Agostini est intéressant car il révèle les difficultés des ces immigrés : à l'annonce du chantier de réunion des Tuileries au Louvre en 1855, le sculpteur d'ornements Martrou lui rédige une lettre de recommandation rappelant « sa compétence dans le travail du marbre » et précisant qu'il se trouve actuellement au chômage, " ses collègues ne le comprenant pas » (16 mars, Archiv. nat. : 64AJ 176 et 64AJ 188). En 1880, on retrouve son nom rajouté au crayon dans la «Liste des sculpteurs d'ornements à employer aux travaux d'architecture du nouvel Hôtel de Ville » avec la mention «sculpteur ouvrier, en parler à M. Villeminot ». Cette référence à Villeminot s'explique par l'habitude qu'avait cet entrepreneur d'ornements d'employer des italiens : ainsi à l'Hôtel de Ville, il avait engagé MM. Viola, Zani, Miclano, Maffio et le mouleur Francesconi (Archiv. Paris : V. $1 \mathrm{M}^{2} 29$ ). En le plaçant dans cet atelier, l'administration trouvait une solution à sa mauvaise compréhension du français.

Il est apparu au cours de la recherche que ce travail de « pratique » était une étape obligatoire de la formation des sculpteurs. Certains s'y stabilisaient, d'autres poursuivaient leur ascension professionnelle. Au Louvre, le chef d'atelier Lafrance retrace l'évolution professionnelle d'un de ses jeunes collaborateurs M. Laurent :

« assidu à son travail, de conduite remarquablement bonne, il est entré sous ma direction au commencement de 1845 comme tailleur de pierres sortant du chantier de M. Casier. Intelligent, je lui ai démontré la mise au point. Il l'a saisie, apprise très promptement et l'a mise en pratique avec une grande précision. Ce progrès de sa part m'a permis d'employer ce bon ouvrier à la grande frise d'attique de la Bibliothèque, à la grande console accotoir du pavillon Mollien, à la rosace arêtière de la galerie de la Bibliothèque, au fronton des croisées de la cour du Grand Ecuyer. L'intelligence éprouvée et justifiée du sieur Laurent l'a conduit à participer à plusieurs statues décorant le palais. Il peut être mis au rang des premiers metteurs au point » (Arch. nat. : 64AJ 205).

On suit ici l'artisan qui passe de la taille, à la mise aux points, puis à la pratique de motifs de plus en plus importants avant d'être responsable de celle d'un fronton.

Interviennent également les sculpteurs d'ornements qui « exécutent toutes les parties accessoires dites d'ornements d'architecture » : leur travail comprend les travaux d'estampage, de restauration (reprises, grattage, raccords) et bien sûr l'exécution des ornements et des petites figures.

Les ornemanistes (ou ornementistes) exécutent toutes sortes de petits motifs végétaux ou floraux, des figures géométriques et des arabesques qu'ils combinent de façon répétitive, symétrique ou alternée pour les frises, rinceaux, moulures, panneaux, cartouches etc. 
Leur apprentissage en atelier (souvent familial) leur a permis de se familiariser à la pratique des savoir-faire traditionnels, des innombrables motifs en cours et des divers matériaux : il n'est donc pas rare de voir un sculpteur exécuter la décoration extérieure sur pierre des façades puis continuer avec les stucs, les staffs et terminer par des lambris en bois. La variété de leurs compétences leur permet de passer indifféremment d'une matière à l'autre, alternant les pratiques avec un même brio, quitte à s'engager dans un atelier industriel ou une entreprise de sculpture d'édition lorsque la construction se fait plus rare. Les ornemanistes candidats aux travaux du Louvre comme plus tard à ceux de l'Hôtel de Ville ne spécifient que rarement s'ils souhaitent une part de travail sur pierre ou sur bois. Ainsi, Edouard Charrier qui a travaillé dès 1849 à la restauration de motifs en pierre des façades du Vieux Louvre finira sa dernière intervention sur le chantier en 1859 avec la « porte en noyer sous la descente à couvert du ministère d'Etat » (ordre no 2, 20 janvier, Arch. nat. : 64AJ 80).

Les dessins d'ornement « à grandeur d'exécution » sont reportés à l' aide de calque en papier, en métal ou en bois (cf. Lettre de l'ornemaniste Thelen à Lefuel : «J'ai reçu le calque d'une fenêtre de la façade du nouveau Louvre que vous m'avez adressé le 3 courant », s. d. [ca mai 1854], Ibidem, 64AJ 188). Pour marquer la pierre, ces calques sont perforés de petits trous qui suivent le tracé du motif : on tamponne alors ce poncif d'une poudre colorée afin d'en reproduire le dessin en pointillé. Le sculpteur peut aussi tracer directement le motif d'un simple trait de ciseau (certains sont d'ailleurs encore visibles sur les parties hautes au Louvre). Si la majorité de ces ornemanistes reçoit de l'administration le dessin de son motif ou le modèle en plâtre, certains, reconnus pour leur compétence, s'en voient confier le modèle et l'exécution (ordre ${ }^{\circ} 116$ envoyé le 2 mai 1856 à M. Cousseau, Ibid., 64AJ 80). Ils en assurent l'entière réalisation ou la partagent avec d'autres exécutants dont ils surveillent l'exactitude du travail (cf. Rouillard chargé du modèle des chapiteaux du manège de l'Empereur, d'une partie de leur exécution et de la surveillance de l'ensemble, Ibid., $\left.\mathrm{F}^{21} 1758\right)$.

Par contre pour les ornements figurés, on fait appel à un figuriste : étymologiquement, ce sculpteur est un mouleur « qui coule des figures en plâtre » (cf. Dictionnaire de l'Académie française ou Littré). Il ne faut donc jamais oublier que cette profession est issue du moulage dont elle a hérité l'art de travailler une matière fine et souple qui permet toutes sortes de précisions et de nuances : sur les abeilles du pavillon Richelieu, les détails des ailes et des pattes sont bien visibles du sol et les chiens de la cour Napoléon confirment des connaissances précises d'anatomie canine. La finesse de ces petits motifs nécessitant soin, minutie et dextérité, les figuristes sont reconnus comme les sculpteurs d'ornements les plus habiles. L'étude de la composition des ateliers du Louvre atteste que c'est dans leurs rangs que sont choisis les chefs d'équipe (Archiv. nat., 64AJ 176).

Ces sculpteurs conservent longtemps l'habitude de travailler de petits formats (comme des plâtres). Cependant au cours du siècle, une évolution a pu être notée : comme le terme traditionnel de figure définit « la forme extérieure d'un corps », les premiers figuristes exécutent des visages (frises, mascarons, cartouches, rosaces) puis de petits personnages (moulures, frises, clefs). Peu à peu, ils étendent leur savoir-faire aux animaux (frises, consoles, tympans). Enfin, par extension, ils illustrent des repré- 
sentations symboliques comme une vertu (La Tempérance), une science (La Géographie), ou une ville (Rouen).

On ne peut qu'être frappé du nombre de ces figures sur les façades du nouveau Louvre : têtes joufflues, mascarons et chérubins nichent dans les clefs, génies musclés porteurs des palmes impériales se cachent entre les œils-de-bœuf, enfants hybrides, sirènes ou satyres s'incurvent à l'angle des attiques ou s'exposent sur les panneaux ou cartouches tandis que toutes sortes d'animaux animent les frises ou complètent les compositions. Lorsqu'elles sont « en pied », les figuristes les travaillent en rondebosse : souvent placés en haut des édifices, debout, les corps dénudés sont déformés pour atténuer les effets de la perspective. Une comparaison entre les différents groupes de génies au Louvre, à l'Opéra et à l'Hôtel de Ville fait apparaître des constantes comme le traitement excessif de la tête, le soin de la gestuelle corporelle et la lisibilité graphique des attributs censés donner leur signification allégorique aux personnages.

Leur dextérité les spécialise parfois dans un type d'ornement ou un style particuliers : au Louvre, Fouché, Gœury et Margotin ne font que des « vermicules aux chaînes d'angle » (Ibidem, 64AJ 80), au Grand Palais, Dupuy et Corbel exécutent plus de « 220 cannelures sous les chapiteaux du porche central » (Ibid., F ${ }^{12} 4069$ B et F ${ }^{12} 4156$ ). D'autres revendiquent une spécialité stylistique : Daillion envoie sa candidature

« en (sa) qualité de sculpteur ornemaniste aux Édifices diocésains : ayant travaillé et fait de profondes études dans l'art de la sculpture, surtout le gothique qui m'est très connu, je me recommande de toute ma force à Monsieur le Ministre, je le prie de venir en aide au pauvre artiste qui, depuis longtemps, est sans travaux » (14 juin 1850, Ibid., $\mathrm{F}^{19}$ 4543).

Damien, lui, en 1856, vient de terminer un travail important « destiné à l'église paroissiale de Mamers (...) dans un style du $\mathrm{XV}^{\mathrm{e}}$ siècle : mes études spéciales du Moyen Âge me permettent d'entreprendre aussi bien l'ornementation que les figures, et de soigner autant l'une que l'autre » (Ibid.). Rambert qui a travaillé à l'hôtel Cluny se fait recommander par Du Sommerard qui confirme ses compétences Renaissance (Ibid.). Louis Gand, lui, «s'est spécialement occupé des styles des $\mathrm{XII}^{\mathrm{e}}$ au XVI ${ }^{\mathrm{e}}$ siècles (et) a travaillé aux restaurations de plusieurs cathédrales », quant à Marneuf, il avait postulé en 1832 aux travaux des Bâtiments civils, fort de son « adresse dans les XVII ${ }^{\mathrm{e}}$ et XVIII ${ }^{\mathrm{e}}$ siècles » (Ibid., $\mathrm{F}^{13}$ 642)

Cette spécialisation progressive est illustrée par un homme comme Pierre Rouillard formé initialement à l'ornement (Tuileries, 1849-50, Arch. nat. : $\mathrm{F}^{21} 1758$ ). Devenu chef d'atelier au Louvre, il acquiert une certaine habileté dans la figure (1855-1856, Ibidem, $\mathrm{F}^{21} 1758$ et $64 \mathrm{AJ}$ 80) puis dans les animaux (chapiteaux du manège du Louvre, 1860, Ibid.). Á l'Opéra, il passe à la statuaire animalière à très grande échelle (4 grands aigles aux encoignures, 1867, Ibid., $\mathrm{AJ}^{13}$ 547) et voit son art reconnu à l'exposition universelle de 1878 avec la commande du « Cheval » pour le bassin inférieur de la cascade du Trocadéro, (Ibid., $\mathrm{F}^{12} 3225$ et $\mathrm{F}^{12}$ 3532).

Contrairement, à ce que certains ont craint, l'expansion des ateliers de moulage de « pierre recomposée » n'a pas totalement étouffé le travail des ornemanistes. Si des entreprises de maçonnerie se révélaient aptes à reproduire et à fabriquer en série des motifs moulés, elles restaient incompétentes pour l'exécution de motifs particuliers. 
Ainsi Krantz, le directeur des travaux de l'exposition universelle de 1878, est obligé de préciser le 16 avril que dans le marché de maçonnerie signé avec l'entreprise Masselin et $\mathrm{C}^{\mathrm{ie}}$, « le prix ne comportait pas les sculptures d'ornement qui ne peuvent être faites que par un artiste spécial ». C'est donc à un sculpteur d'ornements, Villeminot, que sera confié ce travail (Arch. nat. : $\mathrm{F}^{12} 3532$ ).

L'élément sculpté achevé en atelier doit être alors acheminé vers la façade qu'il doit décorer : les différents cahiers des charges précisent que c'est l'administration qui en assure la dépense. On a vu qu'un praticien pouvait travailler sur plusieurs statues d'un même édifice : Bergerat avait chez lui simultanément les pierres des statuaires Chervet, Demesmay, Dumilâtre et Le Père (Arch. Paris : V.1M $\mathrm{M}^{2} 31, \mathrm{~V} .1 \mathrm{M}^{2} 32$ et $\mathrm{V} .1 \mathrm{M}^{2} 35$ ). Il était donc important qu'elles soient évacuées au fur et à mesure de leur achèvement. Cependant, si les ateliers demandaient à être libérer des statues terminées, l'agence des travaux devait gérer le stockage de ces pièces sans encombrer le chantier. Au Louvre, pour éviter ce genre de désagrément, Lefuel tente d'améliorer l'aménagement de hangars où sont disposées les statues en attente de leur installation définitive. D'autres séries d'archives font état de transfert dans les hangars du Dépôt des marbres à l'Île aux Cygnes : elles y sont entreposées dans l'ordre inverse à celui dans lequel elles seront sorties pour être mises en place. Ce travail est confié à des entreprises spécialisées d'emballeur ou de layetier. Les entreprises Cotel et Poupinel sont actives vers 1850, la maison Chalet, successeur de Dubrujeaud à l'exposition universelle de 1878 (Arch. nat. : $\mathrm{F}^{12}$ 3532), et celle de Paul Butet, ancienne maison Autin et Clerc à celle de 1900 (Ibidem, $\mathrm{F}^{21} 4069$ et $\mathrm{F}^{21}$ 4074).

La dernière étape du travail de sculpture consiste en la mise en place et au « parement ». La commande de grands formats ou d'un lot d'ornements composites était souvent répartie en plusieurs parts par souci d'économie, de rapidité d'exécution et de facilité de transport. D'autre part, la fragmentation par petits lots d'un ensemble important permettait de pourvoir une majorité d'ouvriers mais il nécessitait à terme un travail d'assemblage : praticiens, ornemanistes, figuristes et animaliers étaient alors rappelés sur le chantier. L'agencement de ces motifs exécutés en dehors de leur contexte provoquait parfois des litiges que l'administration devait arbitrer. Au Louvre, une plainte du sculpteur Émile Fouquet contre son chef d'atelier fait allusion à ce genre de difficulté :

«le 28 décembre 1850, j'ai reçu une lettre de M. Bergadieu, délégué aux travaux de sculpture du Louvre, qui me disait de commencer une frise au Louvre. Cette lettre est le premier avis que j'ai reçu, j'ai commencé immédiatement. Le modèle étant plus long M. Bergadieu me dit que je supprime un des bouts, que c'était l'ordre que vous lui aviez donné. M'étant aperçu après douze jours de travail que cette frise ne pouvait aller, j'ai prévenu M. Bergadieu que je suspendais ». Or, il est noté en rouge dans la marge : «M. Fouquet sculpteur de cet atelier mis en œuvre par M. Bergadieu pour l'exécution d'une frise sur le modèle de laquelle il a supprimé à l'exécution un des bouts, le modèle se trouvant trop long, ce qui a occasionné la suppression de la sculpture faite et une mise de pierre. Demande une indemnité de son travail de 12 jours, n'ayant dit-il agi pour cette suppression que par ordre de M. Bergadieu » (Arch. nat. : 64AJ 176). 
Des ajustements sont donc nécessaires, des joints retravaillés, des raccords refaits et des mémoires déduits.

Une fois scellée au ciment sur la façade, il reste à achever le parement de la surface de la pierre. Il importe effectivement d'uniformiser les finitions et le polissage des différents éléments d'un ensemble : c'est l'architecte qui en est seul juge. Ainsi à l'Hôtel de Ville, Jacquemart demande des précisions pour son lion :

«Comme la patine est affaire de goût, Monsieur Ballu est le maitre absolu de la décoration et du parti qu'il veut en tirer. L'avis qui prévaudra sera nécessairement le mien. » (Arch. Paris : V.1 $\mathrm{M}^{2} 33$ )

En fin de chantier, sont apparues plusieurs mentions de graveurs sur pierre, sculpteur-graveur ou graveur en architecture : Dieck, Cottebrune et Aubry interviennent dans les années 1850-1860, Gallois et Poignant entre 1855 et 1865, Sulpis et Tribout vers 1860, puis Admant et Dufrénois dans la décennie suivante (1870-1880).

Les almanachs professionnels distinguent le graveur en architecture du graveur lithographe (qui effectue des impressions mécaniques). Il ne s'agit pas non plus du graveur en caractères (typographe sur acier, poinçons ou timbre) ni du graveur en écriture (sur billets et factures). On a constaté que les noms recensés ne figuraient dans aucune liste de ces « graveurs » mais par contre on les retrouvait dans celles de « sculpteurs d'ornements».

En étudiant leurs papiers à lettres, les en-têtes de leurs mémoires et leurs correspondances, il apparaît que ces hommes travaillant sur pierre, marbre et acier, sont spécialisés dans la gravure d'inscriptions (demandant minutie et adresse : textes lapidaires, ex-voto, armoiries, attributs) et qu'ils manient avec dextérité tous formats et sujets (cf. papier à en-tête d'E. Dufrénois, Arch. Paris : V.1M²32). Les styles les plus variés peuvent leur être demandés : au Trocadéro, en 1878, il est précisé dans les soumissions déposées par Admant et Dufrénois que la « gravure des noms des principaux artistes français dans les tables situées au dessus des baies géminées des 2 ailes [est] à exécuter dans le style François $\mathrm{I}^{\mathrm{er}} \gg$ (18 mars 1878, Arch. nat. : $\left.\mathrm{F}^{12} 3337\right)$.

Sur le socle des statues, ils gravent le nom pour en faciliter l'intelligibilité (cf. commande des noms des saints de la colonnade de La Madeleine, Arch. Paris : V. 36 $\mathrm{M}^{32}$ ). $\mathrm{Au}$ palais du Luxembourg, chacune des statues entourant le fronton du couronnement de la façade sur jardin est nommée : on reconnaît ainsi L'Aurore et La Nuit, La Guerre, La Sagesse, L'Éloquence, La Prudence, La Justice, La Guerre et La Paix. De même au Louvre, pour la balustrade des grands hommes sur la place Napoléon, chacun est désigné (cf. Aubry exécute le travail pour 21 statues, Arch. nat. : $F^{21} 3193$ et Dieck pour 22 statues, Ibidem, 64AJ 189). Payés à la lettre, les graveurs devaient préférer se voir attribuer le nom de Montesquieu à celui de Suger.

On fait également appel à eux pour identifier un bâtiment : les divers pavillons du Louvre portent leur nom sur des cartouches de marbre. Les graveurs marquent aussi la date de la construction et la signature des intervenants (cette habitude commencée en 1830 à Paris verra son apogée dans les années 1890 : architectes, entrepreneurs, constructeurs, statuaires et sculpteurs y sont ainsi signalés au passant).

Sur d'autres façades, sont reportés un texte mémorial ou une devise. Aux guichets du Louvre, une large plaque de marbre rappelle l'œuvre salutaire de l'Empe- 
reur : « Napoléon III Empereur des français / réédifie de 1861 à 1868 l'aile du palais des Tuileries / élevée de 1607 à 1663 par Henri, Louis XIII et Louis XIV ». Lors de la construction des écoles municipales, la nef parisienne est systématiquement légendée du Fluctuat nec mergitur. Quant aux bâtiments commerciaux ou syndicaux, ils affichent les devises de la maison : la Société centrale des architectes fait inscrire en 1899 sur le cartouche dominant la porte du 8 , rue Danton $\left(6^{\mathrm{e}}\right)$ la formule emblématique : Le Beau, Le Vrai, l'Utile.

Enfin, une composition littéraire peut renforcer le côté didactique d'un motif allégorique : Paul Sédille sollicite le sculpteur André Allar en 1895 pour l'immeuble de rapport de $\mathrm{M}^{\text {mes }}$ Daligny et Gallin au 30, rue Galilée (16 $\left.{ }^{\mathrm{e}}\right)$. Suivant les dessins de l'architecte, le statuaire exécute deux élégantes cariatides pour encadrer la porte de l'immeuble de rapport. Or, sur la partie horizontale du linteau de la porte, un quatrain explicatif de ces deux figures a été gravé :

«Lasse des vains espoirs et des bruits de la terre / Heureuse d'oublier, l'une a fermé les yeux / A son premier matin, sans effroi ni mystère / L'autre aspire à la vie en souriant aux cieux. »

Ces différentes fonctions de l'écriture en architecture peuvent se combiner entre elles sur une même façade comme sur la façade principale de l'Opéra où des inscriptions précisent les arts célébrés dans cette nouvelle salle de spectacles (l'avant-corps de droite porte la mention Poésie Lyrique). Un autre cartouche explique le thème allégorique de chaque fronton (Ibidem, Sculpture Peinture apposée sur un écusson). Enfin, on peut lire la signature du statuaire des frontons (Ibidem, Gruyère visible sur le rouleau tenu par le génie de droite).

Cette longue analyse des sources a permis de préciser le travail de chacun, son rôle et ses relations avec les autres partenaires du chantier. On a pu également constater que si tous les sculpteurs de façades commençaient par un travail de taille, certains avaient évolué au cours de leur carrière vers la mise aux points, la pratique, l'ornement, la gravure et pour les plus talentueux vers la statuaire. Cette progression est à l'image de la hiérarchie qui régit la profession et les équipes sur un chantier. Les nombreuses spécialités révèlent un souci d'excellence dans l'exécution et une dextérité qui font écho au passage obligatoire dans les divers ateliers d'art décoratif auquel était tenu tout jeune sculpteur d'ornements en formation (cf. Livret-Annuaire, 2005-2006). 